\title{
TOPOLOGICAL ORBIFOLDS
}

\author{
CARLA FARSI
}

(Communicated by Palle E. T. Jorgensen)

\begin{abstract}
We show that two topologically homeomorphic orbifolds are also Lipshitz homeomorphic. We then prove that the $L$-class of a good orbifold with finite fundamental group depends only on the topological structure.
\end{abstract}

\section{LIPSCHITZ STRUCTURES}

A $q$-dimensional smooth orbifold $Q$ is a second countable topological space endowed with an atlas $\mathbf{U}=\left\{U_{i}, \phi_{i}\right\}_{i \in I}$ of open sets, with each $U_{i}$ homeomorphic to $\mathbf{R}^{q} / G_{i}$, where $G_{i}$ is a finite subgroup of $\mathbf{O}(q)$. Let $\phi_{i}: U_{i} \mapsto \mathbf{R}^{q} / G_{i}$ be such a homeomorphism. The following compatibility condition must also be satisfied: $\forall i, j$ for which $U_{i} \cap U_{j} \neq \varnothing, \phi_{j} \circ \phi_{i}^{-1}$ locally lift to a diffeomorphism from $\mathbf{R}^{q}$ to $\mathbf{R}^{q}$.

A smooth manifold is trivially an orbifold. (Take $G_{i}=1 \forall i$.) Let $Q$ be a smooth orbifold. If $x \in Q$, we define $G_{x}$ to be the finite subgroup of $\mathbf{O}(q)$ for which $Q$ looks like $\mathbf{R}^{q} / G_{x}$ arbitrarily close to $x$. If $x, y \in Q, x \sim y$ if and only if $G_{x}$ is conjugate in $\mathbf{O}(q)$ to $G_{y}$. The equivalence classes of points of $Q$ are called the strata of $Q$. If $x \in Q$ with $G_{x} \neq 0, x$ is said to be singular and the set of singular points is called the singular set.

Proposition 1. The following are equivalent:

(1) $Q$ is a smooth orbifold.

(2) $Q$ is the quotient of a smooth locally free action by a compact Lie group $G$ on a smooth manifold $P$.

Proof. See [5, 1]. For any orbifold $Q$ we construct the orthonormal frame bundle $P$ of $Q$ on which the $\mathbf{O}(q)$-action is locally free. Conversely we use the slice theorem to give a local description of the action of $G$ on $P$.

Definition 2. A topological orbifold $Q$ is a second countable topological space endowed with an atlas $\mathbf{U}=\left\{U_{i}, \phi_{i}\right\}_{i \in I}$ of open sets, with each $U_{i}$ homeomorphic to $\mathbf{R}^{q} / G_{i}$, where $G_{i}$ is a finite subgroup of $\mathbf{O}(q)$. Let $\phi_{i}: U_{i} \mapsto \mathbf{R}^{q} / G_{i}$ be such a homeomorphism. The following compatibility condition must also

Received by the editors September 25, 1991.

1991 Mathematics Subject Classification. Primary 46L80, 57S25. 
be satisfied: $\forall i, j$ for which $U_{i} \cap U_{j} \neq \varnothing, \phi_{j} \circ \phi_{i}^{-1}$ locally lift to a homeomorphism from $\mathbf{R}^{q}$ to $\mathbf{R}^{q}$. (If we require that $\phi_{j} \circ \phi_{i}^{-1}$ locally lift to a Lipschitz homeomorphism, we obtain a Lipschitz orbifold.)

As above, a stratification can be defined on the topological orbifold $Q$. It is a classical result of Sullivan that topological manifolds admit a unique Lipschitz structure. Rothenberg and Weinberger generalized Sullivan's theorem to topological manifolds with certain group actions (see [7]; for our purposes (A) is enough) as stated in [6]:

Theorem 3. Let $M$ be a topological manifold on which the finite group $G$ acts continuously and locally linearly, with no component of $M^{H}$ of dimension four for every subgroup $H$ of $G$. Then $M$ admits a $G$-invariant Lipschitz structure (unique up to Lipschitz conjugacy at least when $G$ has odd order).

Definition 4. An orbifold homeomorphism between two topological orbifolds $Q_{1}$ and $Q_{2}, f: Q_{1} \rightarrow Q_{2}$, is a homeomorphism between the two underlying spaces which preserves strata.

If $Q_{1}$ and $Q_{2}$ are as in Definition 4, they will be said to be orbifold homeomorphic.

Theorem 5. Two orbifold homeomorphic topological orbifolds with no strata of dimension four are Lipschitz homeomorphic.

Proof. This is an adaptation of the proof in [7]. We will prove the existence of a Lipschitz structure on an orbifold $Q$. In an analogous way we can argue for the existence of a Lipschitz homeomorphism between two orbifolds $Q_{1}$ and $Q_{2}$ noticing that we are going to approximate the homeomorphism $f: Q_{1} \rightarrow Q_{2}$. We induce on the number of strata and their size. Start with the one with the smallest stabilizer $G_{x}=1$. This is an open dense set in $Q$, and we have on it the Lipschitz structure induced by the orbifold charts $U_{i}$. (This stratum is a Lipschitz manifold.) To extend the Lipschitz structure to the next stratum, we write it locally as a quotient $\widetilde{U} / G_{y}$ and put on $\widetilde{U}$ the Lipschitz structure induced by the one we already have. The action of $G_{y}$ can be made Lipschitz on it by Theorem 3. Since the Lipschitz structures must coincide on an open dense set each time, they must agree. To illustrate the procedure we just described we take the orbifold whose underlying space is a triangle $T$. The inside of $T$ has the smallest stabilizer $G_{x}=1$, while on the edges the stabilizers are $\mathbf{Z}_{2}$, and at the vertices dihedral groups. A neighborhood $U$ of the base $b$ of $T$ can be obtained as the $\mathbf{Z}_{2}$-quotient of an open neighborhood $\widetilde{U}$ ( $\widetilde{U}$ folds along $b$ to give $U$ as quotient). We put on $\widetilde{U}$ the unique Lipschitz structure existing on $\operatorname{Int}(T) \cup \widetilde{U}$. The action of $\mathbf{Z}_{2}$ can be made Lipschitz by Theorem 3. Then we redo the same with the three vertices of $T$.

\section{THE $L$-CLASS}

Now suppose that $Q=P / G$ is a $C^{\infty}$ even-dimensional orbifold. We want to investigate the dependence of the $L$-class (i.e., the class in $K K$-theory of the signature operator) of $Q$. If $M$ is a smooth manifold then a theorem of Hilsum guarantees that the class of the signature operator on $M$ depends only on the Lipschitz structure and, therefore, only on the topological structure of $M$. 
Theorem 6 (Hilsum [3]). Let $D$ be the signature operator on a closed Lipschitz manifold $M$ and let $K_{g}$ be the Hilbert space of forms $\omega$ such that $\int \omega \wedge$ $* \bar{\omega}<+\infty$ with the hermitian product $\left(\omega_{1}, \omega_{2}\right)=\int \omega_{1} \wedge * \bar{\omega}_{2}$ with respect to a Riemannian structure $g$ on $M$. Then

(i) $D$ is a selfadjoint operator on $K_{g}$;

(ii) $[D, \phi]$ is bounded for every Lipschitz function $\phi$ on $M$;

(iii) $\left(1+D^{2}\right)^{-1}$ is compact; and

(iv) the pair $\left(K_{g}, D\left(1+D^{2}\right)^{-1 / 2}\right)$ defines an element in $K_{0}(M)$ whose class does not depend on the choice of $g$, but only on the Lipschitz structure of $M$.

In analogy with the manifold case we (see $[1,2])$ have the following:

Definition 7. The $L$-class of a closed smooth orientable even-dimensional orbifold $Q$ is the class $[\widetilde{S}]$ of the signature operator $S$ on $Q:[\widetilde{S}] \in K K\left(C^{*}(Q), \mathbf{C}\right)$, where $C^{*}(Q)$ is the $C^{*}$-algebra of the foliation of $P$ by $G$-orbits.

When $Q$ is a smooth manifold, $[\widetilde{S}]$ depends only on the topological structure of $Q$ by Theorem 6. This happens also in the orbifold case, at least when $Q$ is good and its fundamental group is finite. The proof is an equivariant version of Hilsum's result since in this case

$$
[\widetilde{S}] \in K K\left(C^{*}(Q), \mathbf{C}\right) \cong K K_{\Gamma}\left(C_{0}(\widehat{Q}), \mathbf{C}\right)
$$

(cf. [2, Proposition 2]), where $\widehat{Q}$ is the universal cover and $\Gamma$ is the fundamental group of $Q$, and Hilsum's proof can be repeated verbatim, the only change being that $\Gamma$ acts everywhere.

Theorem 8. Let $[\widetilde{S}]$ be the L-class of a smooth closed good orientable evendimensional orbifold $Q$ with finite fundamental group. Then $[\widetilde{S}]$ depends only on the topological structure of $Q$.

It is also possible to define the $L$-class of a Lipschitz good even-dimensional orbifold $Q$ with finite fundamental group, odd order stabilizers, and all strata of even dimension. In fact, $Q=\widehat{Q} / \Gamma$, with $\widehat{Q}$ a closed oriented Lipschitz manifold and $\Gamma$ a finite group acting locally linearly on $\widehat{Q} . \widehat{Q}$ admits a $\Gamma$-invariant Lipschitz structure. Then the signature operator on $\widehat{Q}$ can be interpreted as an operator on $Q$ and defines (see [4]) an element of $K K_{\Gamma}(C(\widehat{Q}), \mathrm{C})$ which coincides with the one in Definition 7 if $Q$ is smooth. We have (see [4, Proposition 4.6]):

Theorem 9. Let $[E] \in K K_{\Gamma}(\mathbf{C}, C(\widehat{Q}))$. Then the index $\mathscr{S}_{E}$ of the signature operator on $Q$ with coefficients in $E$ is given by (using the notation in [4])

$$
\mathscr{S}_{E}=\frac{1}{|\Gamma|} \sum_{h \in \Gamma}\left\langle\operatorname{ch} i^{*}(E) \omega\left(h, \widehat{Q}^{h}\right) L\left(\widehat{Q}^{h}\right),\left[\widehat{Q}^{h}\right]\right\rangle .
$$

\section{ACKNOWLEDGMENTS}

We thank Professor J. Rosenberg for helpful conversations and the referee for useful comments and suggestions. 


\section{REFERENCES}

1. C. Farsi, K-theoretical index theorems for orbifolds, Quart. J. Math. Oxford 43 (1992), 183-200.

2. $\ldots$ K-theoretical index theorems for good orbifolds, Proc. Amer. Math. Soc. 115 (1992), 769-773.

3. M. Hilsum, Opérateurs de signature sur un variété lipschitzienne et modules de Kasparov non bornés, C. R. Acad. Sci. Paris Sér. I Math. 297 (1983), 39-52.

4. __ Functorialité en $K$-théorie bivariante pour les variétés lipschitziennes, $K$-Theory 3 (1989), 401-440.

5. T. Kawasaki, The signature theorem for V-manifolds, Topology 17 (1978), 75-83.

6. J. Rosenberg and S. Weinberger, Higher G-indices (on smooth and Lipschitz manifolds) and applications, preprint.

7. M. Rothenberg and S. Weinberger, Group actions and equivariant Lipschitz analysis, Bull. Amer. Math. Soc. (N.S.) 17 (1987), 109-112.

Department of Mathematics, University of Colorado, Campus Box 395, Boulder, ColORADO 80309-0395

E-mail address: farsi@euclid.colorado.edu 